\title{
Estudo de Desempenho de Algoritmos de Filtragem Colaborativa para Sistemas de Recomendação Educacionais aplicando Agrupamento Prévio de Usuários com Traços de Personalidade Similares
}

\author{
Janderson Jason B. Aguiar'1, Joseana M. F. R. de Araújoํ, Evandro de B. Costa \\ ${ }^{1}$ Universidade Federal de Campina Grande (UFCG), Campina Grande - PB - Brasil \\ ${ }^{2}$ Universidade Federal de Alagoas (UFAL), Maceió - AL - Brasil \\ jandersonecopin.ufcg.edu.br, joseana@computacao.ufcg.edu.br, evandro@ic.ufal.br
}

\begin{abstract}
Some research on Educational Recommender Systems uses the Trait Theory (Personality Traits - PT) when applying Collaborative Filtering $(C F)$. In this paper, we start from the idea that all CF algorithms implicitly deal with users' PT, and by explicitly grouping users based on these PT, the algorithms' performance can improve. In this research, we conducted an experimental study on CF algorithms' accuracy, applying a previous grouping of users (mostly computing students) based on their PT (Big Five model). With the applied strategy, the results indicated that there is the possibility of improving the accuracy of neighborhood-based CF algorithms in the educational domain.
\end{abstract}

Resumo. Pesquisas têm usado a teoria dos Traços de Personalidade (TP) ao aplicar Filtragem Colaborativa (FC) em Sistemas de Recomendação Educacionais. A ideia levantada nesta pesquisa é que todos os algoritmos de FC lidam, implicitamente, com os TP dos usuários, e, ao agrupar explicitamente os usuários, baseando-se nesses TP, o desempenho dos algoritmos pode melhorar. Neste artigo, é apresentado um estudo experimental sobre a acurácia de algoritmos de FC aplicando um agrupamento prévio dos usuários (maioritariamente discentes de computação), baseando-se em seus TP (modelo Big Five). Os resultados indicaram que, com tal estratégia, no domínio educacional, há a possibilidade de melhoria da acurácia de algoritmos de FC baseados em vizinhança.

\section{Introdução}

A Filtragem Colaborativa (FC) é uma das técnicas mais utilizadas em sistemas de recomendação [Alyari e Navimipour 2018]. Tais sistemas possibilitam que pessoas encontrem conteúdo que efetivamente lhes interessa [Aggarwal 2016]. Mais especificamente, os Sistemas de Recomendação Educacionais (SRE) podem ser usados para recomendar recursos didáticos (a exemplo de artigos científicos e objetos de aprendizagem) atendendo às preferências dos usuários (a exemplo de pesquisadores e estudantes) [Costa, Aguiar e Magalhães 2013] [Gianotti, Cazella e Behar 2019].

Por estar relacionada ao processo de tomada de decisões, alguns pesquisadores — a exemplo de Nunes e Hu (2012) e Tkalčič (2018) — defendem que a personalidade deve ser considerada ao projetar sistemas de recomendação. Segundo Hu e Pu (2011) e Nunes e $\mathrm{Hu}$ (2012), deduz-se que pessoas com personalidade semelhante terão interesses e padrões comportamentais semelhantes. Essa dedução foi base para, a partir 
da popular categoria de FC baseada em vizinhança, criar variações definindo tal vizinhança (usuários mais similares) concernente à personalidade.

Tendo-se em vista a essência dos algoritmos de FC (que realizam predições/recomendações baseadas nas preferências anteriores dos usuários sobre os itens disponíveis), neste artigo defende-se que a personalidade dos usuários é um fator que influencia todos os algoritmos de FC implicitamente (visto que utilizam os likes e/ou ratings atribuídos pelos usuários, cuja atribuição foi implicitamente influenciada pelos seus TP). A partir dessa ideia, uma hipótese é que a qualidade da predição/recomendação não diminui se o "treinamento" de um algoritmo de FC for realizado desconsiderando os "dados de opinião" para os itens (por exemplo: likes ou ratings de 1 a 5) de usuários menos similares quanto à personalidade. Além disso, uma hipótese derivada é que, ao executar um algoritmo de FC desconsiderando os dados dos usuários menos similares, em cada TP, a qualidade da predição tende a melhorar.

Visando-se a contribuir com a área de Sistemas de Recomendação Educacionais, o estudo descrito neste artigo objetivou investigar se o uso de dados explícitos da personalidade dos usuários, no domínio educacional, contribui para melhorar a acurácia dos algoritmos de FC (independentemente da categoria de FC), considerando a "colaboração" (dados de opinião - ratings) de apenas usuários agrupados (clusterized) quanto a seus TP. Portanto, a questão de pesquisa estabelecida foi esta: a acurácia das predições de um algoritmo de FC, no domínio educacional, é afetada se o algoritmo for executado desconsiderando os ratings de usuários não tão similares quanto à personalidade de um usuário-alvo de recomendação? (Se sim, como?).

Além desta seção introdutória, neste artigo, são apresentados conceitos e estudos relacionados na Seção 2; o método aplicado é comentado na Seção 3; os resultados são discutidos na Seção 4; e as últimas considerações são apresentadas na Seção 5.

\section{Fundamentação Teórica e Estudos Relacionados}

A personalidade pode ser definida como um conjunto de características de um indivíduo que influencia exclusivamente suas cognições, emoções, motivações e comportamentos em diversas situações [Nunes e Hu 2012]. O modelo Big Five é uma versão moderna da teoria dos Traços de Personalidade (TP) [Allport e Allport 1921]. Tal modelo define estes como os cinco grandes TP: Abertura (ou Abertura à mudança, ou Abertura à experiência), Realização (ou Conscientização, ou Conscienciosidade), Extroversão, Socialização (ou Amabilidade) e Neuroticismo (ou Instabilidade emocional) [Aguiar 2017] [Jaques e Nunes 2019]. O modelo Big Five é o "mais amplamente usado para descrever como uma pessoa geralmente se envolve com o mundo" [IBM 2019].

É comum as pesquisas utilizarem questionários para identificar os TP das pessoas, a exemplo do NEO-PI-R (Revised NEO-Personality Inventory), com 240 questões de múltipla escolha, e do TIPI (Ten Item Personality Inventory), com 10 itens [Jaques e Nunes 2019]. Todavia, cada vez mais prefere-se identificar os TP dos indivíduos de modo menos intrusivo, havendo pesquisas, a exemplo de Schwartz et al. (2013), que indicaram que os TP possuem correlação com padrões de uso do Facebook. Essa pesquisa foi base para o desenvolvimento de uma antiga ferramenta para identificação dos TP via Facebook - a Five Labs [Five 2015 apud Aguiar, Araújo e Costa 2019] —, além de ser referência para o serviço Watson Personality Insights [IBM 
IX Congresso Brasileiro de Informática na Educação (CBIE 2020)

Anais do XXXI Simpósio Brasileiro de Informática na Educação (SBIE 2020)

2019], que infere características da personalidade de um indivíduo a partir de seus textos.

Em relação à Filtragem Colaborativa $(\mathrm{FC})$, é possível afirmar que esta é uma das técnicas de recomendação mais utilizadas, inclusive em SRE [Laisa et al. 2018] [Moraes e Stiubiener 2019]. Com a FC, são realizadas predições/recomendações de itens não conhecidos por um usuário-alvo, mas que foram avaliados como relevantes por usuários com preferências similares àquele. Para isso, os algoritmos de FC baseiamse em vizinhança (neighborhood-based ou memory-based) ou em um modelo aprendido (model-based) [Aggarwal 2016] [Alyari e Navimipour 2018].

Os algoritmos de FC categorizados como neighborhood-based são bastante populares; com foco no usuário, uma etapa fundamental dessa categoria de FC é a identificação dos usuários mais semelhantes a um usuário-alvo (empregando-se alguma medida de similaridade), sendo comum definir um valor $K$ para o tamanho da vizinhança (UserKNN - User-based K-Nearest Neighbors); alternativamente, é possível focar no item (ItemKNN - Item-based K-Nearest Neighbors). Os algoritmos de FC categorizados como model-based, cada vez mais empregados, consistem em aprender um modelo a partir do conjunto de ratings (usando métodos de machine learning e data mining); um destaque no estado da arte em FC tem sido a utilização de algoritmos baseados em fatoração de matrizes, a exemplo de SVD++ (Singular Value Decomposition Plus Plus) e BMF (Biased Matrix Factorization), com implementações disponíveis na biblioteca MyMediaLite [Gantner et al. 2011].

Dentre as pesquisas que propõem estratégias de recomendação baseadas em personalidade, muitas aplicam FC, a exemplo das pesquisas de Tkalčič et al. (2009), Hu e Pu (2011), Elahi et al. (2013), Fernández-Tobías e Cantador (2014) e Roshchina, Cardiff e Rosso (2015) - nenhuma delas com foco no domínio educacional. Dentre tais grupos de pesquisa, é possível destacar $\mathrm{Hu}$ e $\mathrm{Pu}$, e Tkalčič e seus colaboradores, bastante referenciados, e destacados por Chen, Wu e He (2016) - ao abordarem as pesquisas que usam personalidade na recomendação baseada em $\mathrm{FC}$-, além de destacados recentemente por Alves et al. (2020) - ao comentarem os sistemas de recomendação baseados em personalidade em uma revisão referente à incorporação da personalidade no projeto de interface do usuário. Em Tkalčič et al. (2009) e Hu e Pu (2011), foram elaboradas variações de UserKNN, identificando a vizinhança com base no Big Five.

Embora seja mais comum a realização de estudos incorporando $\mathrm{FC}$ e TP envolvendo algoritmos neighborhood-based, há também os que consideram algoritmos baseados em fatoração de matrizes, a exemplo de Yakhchi et al. (2020) — que usaram um conjunto de dados referente a produtos da Amazon. Em SRE, é possível citar as pesquisas de Salehi (2014) e Shah, Shah e Banerjee (2017), que aplicaram fatoração de matrizes, mas não consideraram TP.

Ainda em relação a estudos relacionados, é adequado comentar que há estudos que consideram o uso de clustering (agrupamento) em FC, a exemplo de Zarzour et al. (2018), que combinaram SVD e K-means, obtendo melhorias na recomendação em relação à métrica RMSE (Root Mean Square Error), no domínio de filmes. Sobre o uso de clusters de indivíduos em SRE, é possível citar a pesquisa de Su et al. (2015) definindo clusters de professores usando um método baseado no K-means. Essas pesquisas não realizaram o agrupamento baseado nos TP dos indivíduos. 
No domínio educacional, as pesquisas de Xia et al. (2014) e Aguiar, Araújo e Costa (2019) são algumas das realizadas no contexto de FC usando TP. Como expõe Aguiar, Araújo e Costa (2019), não é comum encontrar pesquisas, nesse contexto, no domínio educacional. Nas revisões de literatura (estudos secundários) realizadas por Aguiar et al. (2015), Drachsler et al. (2015), Erdt, Fernandez e Rensing (2015), Katarya e Verma (2016), Campos et al. (2017), Ferreira, Vasconcelos e França (2017), KlašnjaMilićević et al. (2017), Morais et al. (2017), Laisa et al. (2018), Reis et al. (2018), Leite et al. (2019), Moraes e Stiubiener (2019) e Pinho et al. (2019), dentre os poucos estudos encontrados referentes a SRE e TP, nenhum focou no objetivo descrito neste artigo.

Uma pesquisa recente em desenvolvimento - não referida nesses estudos secundários analisados - é a de Gianotti, Cazella e Behar (2019), apresentada em um consórcio doutoral, propondo um modelo para integrar os TP dos alunos em um SRE, visando a contribuir para a personalização de recomendações de objetos de aprendizagem. Tal pesquisa de doutorado em andamento evidencia que ainda são recorrentes pesquisas estudando a integração entre TP e SRE.

Nesta seção, e também na anterior, há citações de estudos primários e estudos secundários (que, por sua vez, elencaram estudos primários) relacionados ao estudo apresentado neste artigo. Entretanto, com as buscas realizadas - inclusive no portal de publicações da CEIE (Comissão Especial de Informática na Educação) —, não foram encontradas investigações experimentais referentes ao objetivo descrito na Seção 1 nem no domínio educacional, nem em outros domínios. Assim, julgou-se significante a realização e divulgação do estudo descrito nas seções seguintes. Haja vista a ideia da técnica de FC, destaca-se que este estudo associa-se à ideia de colaboração entre indivíduos, sendo o aspecto colaborativo ressaltado em teorias pedagógicas construtivistas — por exemplo, na teoria da aprendizagem de Vygotsky (1978).

\section{Método}

Para analisar o desempenho referente à acurácia dos algoritmos, tendo em vista o objetivo descrito na Seção 1, foram escolhidos dois algoritmos de cada categoria de FC comentada na Seção 2: os algoritmos SVD++ e BMF (categoria model-based) e os algoritmos UserKNN e ItemKNN (categoria neighborhood-based) - sendo definido $K=5$, assim como em $\mathrm{Hu}$ e $\mathrm{Pu}$ (2011). Utilizou-se a biblioteca MyMediaLite para a execução desses algoritmos. Para avaliar a acurácia, foi utilizada a métrica RMSE, comumente empregada em outras pesquisas - como, por exemplo, em Zarzour et al. (2018) e Yakhchi et al. (2020).

Atentando-se para a ideia de considerar a "colaboração" (dados de opinião ratings) de apenas usuários agrupados quanto a seus $\mathrm{TP}$, foi elaborada uma estratégia de variação aplicada a cada um dos quatro algoritmos de FC. Assim, foi utilizado um processo de clustering (agrupamento) para desconsiderar os ratings de usuários não tão similares quanto à personalidade de um usuário-alvo de recomendação.

Para a criação dos clusters (grupos), a estratégia planejada fundamentou-se no modelo de personalidade utilizado. Como cada um dos fatores do modelo Big Five pode ser caracterizado por adjetivos em dois polos - polo do rótulo ou polo oposto, como apresentado em Jaques e Nunes (2019) —, foram definidos dois clusters por traço. Isto é, para executar determinado algoritmo de FC em relação a determinado usuário-alvo, foram previamente desconsideradas as opiniões (ratings) dos usuários menos similares 
em relação a cada traço; e, por fim, utilizada a média das execuções referentes aos cinco clusters dos quais determinado usuário-alvo participa.

Uma forma de definir os dois clusters, por traço, seria simplesmente agrupar os usuários com valores maiores que 50\%, com os demais no outro cluster. Entretanto, as pessoas, na realidade, não são uniformemente distribuídas quanto aos $\mathrm{TP}$, podendo, inclusive, em uma certa população, estarem todas caracterizadas por valores acima de $50 \%$ em relação a algum(ns) traço(s), mas ainda se diferindo em relação ao nível percentual (mais próximo ou não de 100\%). Assim, decidiu-se usar o algoritmo K-means - aprendizado não supervisionado - para definir os dois clusters por traço. Para identificar cada variação dos algoritmos analisados no experimento (com essa estratégia de agrupamento prévio de usuários com TP similares), foi adicionado o sufixo '_tps': SVD++_tps, BMF_tps, UserKNN_tps e ItemKNN_tps.

Foram utilizados dois conjuntos de dados (datasets) de domínio educacional, nomeados neste artigo como OAs\&DiscentesCC (usado em [Aguiar, Araújo e Costa 2019]) e RecSalt (usado em [Magalhães et al. 2015]), cujos usuários definiram ratings de 1 a 5 para os itens. O dataset OAs\&DiscentesCC envolve 25 itens (representações de objetos de aprendizagem com características variadas do padrão IEEE LOM) e 55 usuários (discentes de computação, em nível de graduação ou pós-graduação), com detecção de personalidade via ferramenta Five Labs. O dataset RecSalt envolve 50 itens (artigos científicos) e 21 usuários (discentes ou diplomados na área da computação, em nível de graduação ou pós-graduação), que responderam ao questionário TIPI, e, dentre estes, 12 usuários usaram a ferramenta Five Labs; portanto, a execução experimental considerou duas variações desse dataset, nomeadas RecSaltTIPI e RecSaltFL.

Ressalta-se que Erdt, Fernandez e Rensing (2015), ao analisarem a literatura relacionada à avaliação em SRE, apontaram que é difícil encontrar datasets, e observaram que o número médio de participantes em estudos com usuários (user studies) foi de 53, com mediana igual a 25, e moda igual a 24.

Para execução experimental e avaliação dos algoritmos, foi utilizado o método Random Subsampling [Han, Kamber e Pei 2012], sendo cada conjunto de dados dividido aleatoriamente, por 50 vezes, em dois subconjuntos: $67 \%$ dos dados para o conjunto de treinamento e $33 \%$ dos dados para o conjunto de teste. Para os intervalos de confiança gerados e para os testes estatísticos realizados (testes T e Mann-Whitney U [Boslaugh e Watters 2008]), foi considerado $\alpha=5 \%$.

\section{Resultados e Discussões}

Baseando-se no método descrito na Seção 3, são apresentados, na Figura 1, os intervalos de confiança (para cada dataset) referentes ao desempenho dos algoritmos em estudo e suas correspondentes variações aplicando agrupamentos (' tps'). Analisandose cada par, foi visto que, com uso do agrupamento baseado em TP, o desempenho de nenhum dos quatro algoritmos diminuiu significativamente (em nenhum dos datasets). Isso reforça a hipótese levantada neste estudo de que, ao executar um algoritmo de FC, sua acurácia no mínimo se mantém ao não dar importância para a opinião dos usuários menos similares quanto à personalidade. A não diminuição dessa acurácia corrobora ainda com a ideia de que todos os algoritmos de FC implicitamente consideram TP (uma vez que analisam ratings definidos por seres caracterizados por TP). 


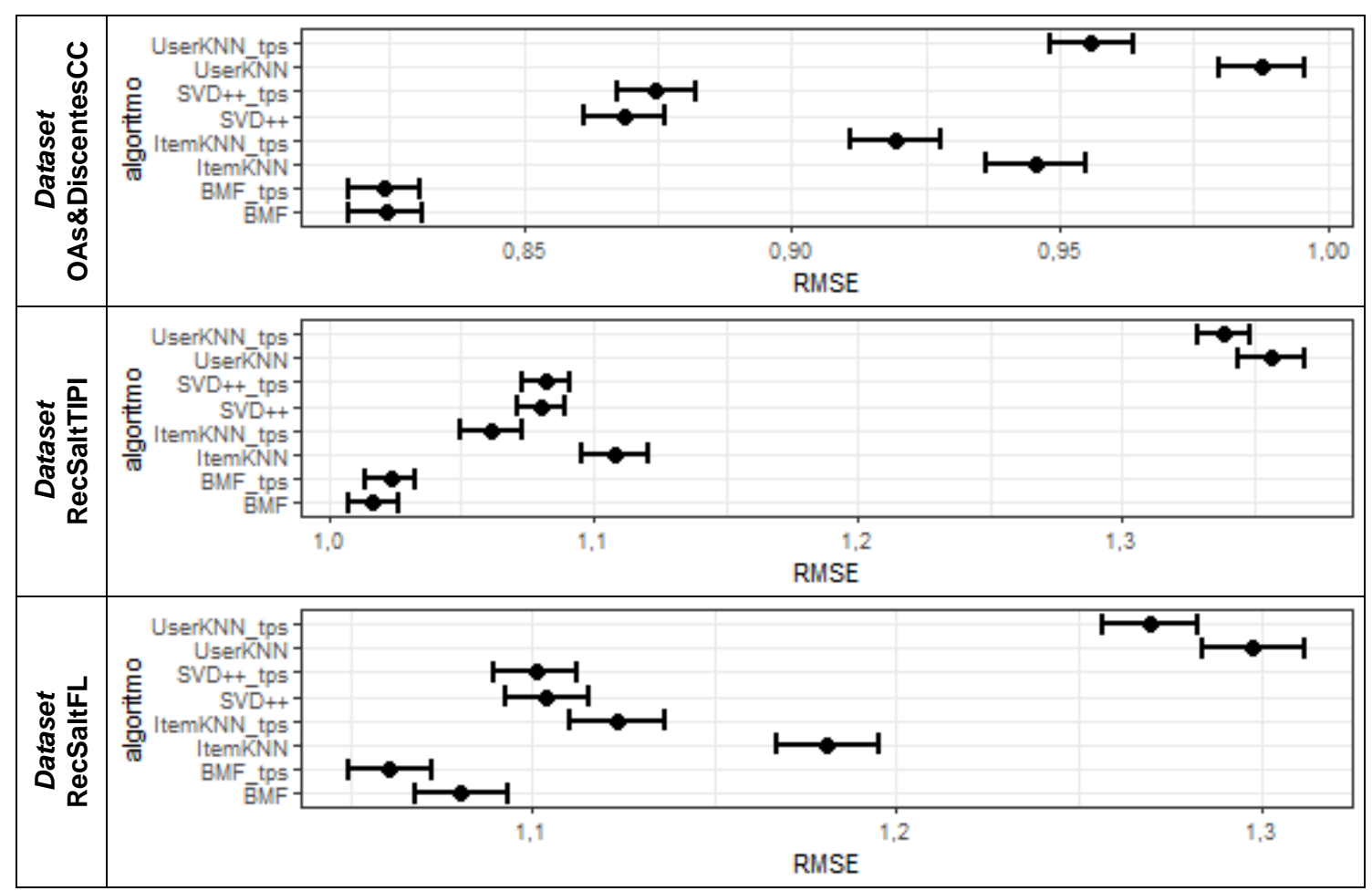

Figura 1. Intervalos de confiança para a média referentes aos datasets utilizados.

Analisando-se o aspecto da acurácia ser afetada, é possível responder positivamente à questão de pesquisa em alguns casos (a exemplo do UserKNN no dataset OAs\&DiscentesCC). No Quadro 1, estão resumidos numericamente os dados da avaliação experimental (média e mediana referentes à métrica RMSE). Na elaboração do Quadro 1, também foi considerado o resultado dos testes T e Mann-Whitney U (para facilitar a análise dos resultados especialmente referentes aos pares de algoritmos cujos intervalos de confiança para a média tiveram alguma sobreposição) — os pares que apresentaram diferença estatística estão com média e mediana destacadas em negrito.

Quadro 1. Dados numéricos do experimento (referentes à métrica RMSE).

\begin{tabular}{|c|c|c|c|c|c|c|}
\hline \multirow{2}{*}{$\begin{array}{l}\text { Categoria } \\
\text { de FC }\end{array}$} & \multirow{2}{*}{$\begin{array}{l}\text { Algoritmo } \\
\text { de FC }\end{array}$} & \multirow[t]{2}{*}{ Dataset } & \multicolumn{2}{|c|}{ Baseline } & \multicolumn{2}{|c|}{$\begin{array}{c}\text { Algoritmo com prévio agrupamento } \\
\text { baseado em TP }\end{array}$} \\
\hline & & & Média & Mediana & Média & Mediana \\
\hline \multirow{6}{*}{$\begin{array}{l}\text { Algoritmos } \\
\text { neighborhood- } \\
\text { based }\end{array}$} & \multirow{3}{*}{ UserKNN } & OAs\&DiscentesCC & 0,9878 & 0,9840 & $0,9560(+3,2 \%)$ & $0,9543(+3,0 \%)$ \\
\hline & & RecSaltTIPI & 1,3563 & 1,3508 & $1,3379(+1,4 \%)$ & $1,3409(+0,7 \%)$ \\
\hline & & RecSaltFL & 1,2979 & 1,3023 & $1,2697(+2,2 \%)$ & $1,2647(+2,9 \%)$ \\
\hline & \multirow{3}{*}{ ItemKNN } & OAs\&DiscentesCC & 0,9456 & 0,9466 & $0,9195(+2,8 \%)$ & $0,9211(+2,7 \%)$ \\
\hline & & RecSaltTIPI & 1,1082 & 1,1106 & $1,0617(+4,2 \%)$ & $1,0652(+4,1 \%)$ \\
\hline & & RecSaltFL & 1,1809 & 1,1771 & $1,1235(+4,9 \%)$ & $1,1242(+4,5 \%)$ \\
\hline \multirow{6}{*}{$\begin{array}{l}\text { Algoritmos } \\
\text { model-based }\end{array}$} & \multirow{3}{*}{ SVD++ } & OAs\&DiscentesCC & 0,8687 & 0,8735 & $0,8747 \quad(-0,7 \%)$ & $0,8811(-0,9 \%)$ \\
\hline & & RecSaltTIPI & 1,0802 & 1,0812 & $1,0820(-0,2 \%)$ & $1,0820(-0,1 \%)$ \\
\hline & & RecSaltFL & 1,1039 & 1,1053 & $1,1009(+0,3 \%)$ & $1,1032(+0,2 \%)$ \\
\hline & \multirow{3}{*}{ BMF } & OAs\&DiscentesCC & 0,8242 & 0,8250 & $0,8238(+0,0 \%)$ & $0,8273(-0,3 \%)$ \\
\hline & & RecSaltTIPI & 1,0170 & 1,0140 & $1,0236(-0,7 \%)$ & $1,0291(-1,5 \%)$ \\
\hline & & RecSaltFL & 1,0805 & 1,0751 & $1,0608(+1,8 \%)$ & $1,0579(+1,6 \%)$ \\
\hline
\end{tabular}

Observação: Os valores entre parênteses são as porcentagens referentes ao aumento do desempenho (ou à redução do desempenho, nos casos de valores negativos) em comparação à abordagem baseline. As diferenças significativas (p-valor < 0,05) são apresentadas em negrito. 
Embora haja diferença no valor numérico da média e da mediana, não foi possível rejeitar a hipótese de que não há diferença de desempenho entre os pares referentes aos algoritmos model-based (SVD++ e SVD++_tps; BMF e BMF_tps), em quaisquer um dos datasets utilizados - p-valores maiores que o nível de significância $\alpha$, tanto para os testes $\mathrm{T}$ quanto Mann-Whitney U. Isso também se observou no par UserKNN e UserKNN_tps para o dataset RecSaltTIPI.

Considerando-se a essência dos algoritmos model-based, é ousado esperar a acurácia não diminuir ao se ter menos dados para a definição interna do modelo; mesmo assim, a acurácia se manteve, reforçando a ideia de que desconsiderar a opinião daqueles menos similares quanto à personalidade não impacta negativamente nos resultados de qualquer algoritmo de FC. Ademais, pensando-se em outros estudos referentes à personalidade em SRE, pode-se ter ganhos em aspectos como explainability (por exemplo: explicar a um aluno/professor/gestor/pesquisador o motivo de uma recomendação fornecida a ele, gerada essencialmente via fatoração de matrizes, provavelmente será menos fácil que se comentar que a recomendação foi definida baseando-se na opinião de pessoas com personalidade similar a ele).

Em relação aos algoritmos neighborhood-based, notou-se que, apesar de haver o caso de não se ter diferença estatística entre UserKNN e UserKNN_tps no dataset RecSaltTIPI, houve, no restante dos casos, melhoria ao utilizar a abordagem de agrupamento baseado em TP, sendo os maiores ganhos referentes ao ItemKNN.

Embora o foco desta pesquisa não seja analisar qual algoritmo de $\mathrm{FC}$ possui o melhor desempenho, adicionalmente observou-se que o algoritmo BMF (e sua variação BMF_tps) proporcionou os melhores desempenhos com os datasets utilizados. Ressaltase que, em SRE, é importante aplicar FC com outras técnicas que atendam à finalidade educacional de determinado sistema de recomendação (por exemplo, considerando o conteúdo dos itens - artigos científicos, objetos de aprendizagem etc.).

\section{Considerações Finais}

Em síntese, a ideia destacada neste artigo é que todos os algoritmos de FC implicitamente lidam com os TP dos usuários e, ao dividi-los em grupos baseados explicitamente nesses TP, hipotetizou-se que o desempenho referente à acurácia dos algoritmos não seria prejudicado - e a aplicação desses agrupamentos tenderia a melhorar tal acurácia. Ao avaliar isso experimentalmente no domínio educacional, visando a responder à questão de pesquisa exposta na Seção 1, os resultados obtidos indicaram, primeiramente, que, de fato, a acurácia de nenhum dos algoritmos de FC diminuiu significativamente. Sobre melhoria, não se observou nos algoritmos model-based; contudo, observou-se a tendência de melhoria nos algoritmos neighborhood-based.

Como estudo futuro, pretende-se avaliar melhorias ao ponderar os traços do modelo Big Five, uma vez que, no domínio educacional, alguns podem ter mais influência, como indiciado em Aguiar, Araújo e Costa (2019). Além disso, é conveniente aprofundar este estudo utilizando outros datasets, de modo a reafirmar os resultados obtidos, uma vez que o número de usuários nos datasets utilizados não é tão expressivo, sendo uma ameaça à validade e limitação do estudo.

Em virtude do uso de questionários dificultar a aplicabilidade de SRE no mundo real, é apropriado expor que Ferreira et al. (2020) exploraram o uso de processamento 
de linguagem natural para detecção automática dos TP a partir de textos em língua portuguesa (do Brasil) - eles ressaltam que a maioria dos trabalhos referentes a esse aspecto é para a língua inglesa. Por exemplo, o Watson Personality Insights (citado na Seção 2), que poderia ser um serviço facilitador para pesquisas brasileiras, ainda não analisa textos em língua portuguesa. Destaca-se, portanto, que as pesquisas em SRE envolvendo TP, em âmbito brasileiro, podem se beneficiar ainda mais com os avanços na detecção automática de personalidade, motivando ainda mais pesquisadores para essa área, pois, como apontam Jaques e Nunes (2019), ainda são poucos.

Por fim, compreende-se que este artigo contribui para a área de Informática na Educação ao apresentar um estudo envolvendo a aplicação da teoria dos TP, em algoritmos usuais para recomendação de recursos educacionais, visando a melhorar soluções referentes à personalização de ambientes computacionais para a promoção da aprendizagem. Ressalta-se que, segundo alguns pesquisadores [Elahi et al. 2013] [Xia et al. 2014], os sistemas baseados em personalidade possibilitam maior eficácia e lealdade, de seus usuários, que aqueles não baseados em personalidade. Reforça-se também que, além de considerar TP e FC em SRE, é importante que o processo de recomendação englobe outras técnicas e aspectos, para atender às particularidades do domínio educacional.

\section{Agradecimentos}

A Jonathas Magalhães e aos participantes de sua pesquisa, pelo apoio referente aos dados.

\section{Referências}

Aggarwal, C. C. (2016). Recommender Systems: The Textbook. 1st ed. Springer International Publishing, 498 p. ISBN: 978-3-319-29659-3.

Aguiar, J. J. B. (2017). Considerando Estilos de Aprendizagem, Emoções e Personalidade em Informática na Educação. Informática na Educação: teoria \& prática, Porto Alegre, v. 20, n. 2, p. 85-102.

Aguiar, J. J. B., Santos, S. I. N., Fechine, J. M. e Costa, E. (2015). Avaliação de Sistemas de Recomendação Educacionais no Brasil: uma revisão sistemática da literatura. In: Anais do Simpósio Brasileiro de Informática na Educação (SBIE), Maceió, p. 1255-1264.

Aguiar, J. J. B., Araújo, J. M. F. R. e Costa, E. B. (2019). Experimentando a Influência dos Traços de Personalidade do Modelo Big Five na Recomendação de Recursos Educacionais. In: Anais do Simpósio Brasileiro de Informática na Educação (SBIE), Brasília, p. 1711-1720.

Allport, F. H. e Allport, G. W. (1921). Personality Traits: Their Classification and Measurement. The Journal of Abnormal and Social Psychology, v. 16, n. 1, p. 6-40.

Alves, T., et al. (2020). Incorporating personality in user interface design: A review. Personality and Individual Differences, v. 155.

Alyari, F. e Navimipour, N. J. (2018). Recommender systems: A systematic review of the state of the art literature and suggestions for future research. Kybernetes, v. 47, n. 5, p. 985-1017.

Boslaugh, S. e Watters, P. A. (2008). Statistics in a Nutshell. 1st ed. O'Reilly Media, Inc., Sebastopol, CA, USA. ISBN: 978-0-596-51049-7.

Campos, A., et al. (2017). Mapeamento de Soluções tecnológicas em Sistemas de Recomendação Educacionais em âmbito brasileiro. Informática na Educação: teoria \& prática, Porto Alegre, v. 20, n. 3, p. 79-96.

Chen, L., Wu, W. e He, L. (2016). Personality and Recommendation Diversity. In: Tkalčič, M., 
IX Congresso Brasileiro de Informática na Educação (CBIE 2020)

Anais do XXXI Simpósio Brasileiro de Informática na Educação (SBIE 2020)

et al. (Eds.), Emotions and Personality in Personalized Services. Human-Computer Interaction Series. Springer, p. 201-225.

Costa, E., Aguiar, J. e Magalhães, J. (2013). Sistemas de Recomendação de Recursos Educacionais: conceitos, técnicas e aplicações. In: Anais da Jornada de Atualização em Informática na Educação (JAIE), p. 57-78.

Drachsler, H., et al. (2015). Panorama of Recommender Systems to Support Learning. In: Ricci, F., et al. (Eds.), Recommender Systems Handbook, p. 421-451.

Elahi, M., et al. (2013). Personality-Based Active Learning for Collaborative Filtering Recommender Systems. In: Baldoni, M., et al. (Eds.), Proceeding of the XIII International Conference on AI*IA 2013: Advances in Artificial Intelligence, v. 8249, p. 360-371.

Erdt, M., Fernandez, A. e Rensing, C. (2015). Evaluating Recommender Systems for Technology Enhanced Learning: A Quantitative Survey. IEEE Transactions on Learning Technologies, v. 8, n. 4, p. 326-344.

Fernández-Tobías, I. e Cantador, I. (2014). Personality-Aware Collaborative Filtering: An Empirical Study in Multiple Domains with Facebook Data. In: Proceedings of the 15th International Conference on Electronic Commerce and Web Technologies, Springer, p. 125-137.

Ferreira, T. B., et al. (2020). Regras para formação de grupos de colaboração utilizando detecção automática de traços de personalidade. Brazilian Journal of Computers in Education, v. 28, p. 273-296.

Ferreira, V. A. S., Vasconcelos, G. C. e França, R. S. (2017). Mapeamento Sistemático sobre Sistemas de Recomendações Educacionais. In: Anais do Simpósio Brasileiro de Informática na Educação (SBIE), Recife, p. 253-262.

Gantner, Z., et al. (2011). MyMediaLite: A Free Recommender System Library. In: Proceedings of the Fifth ACM Conference on Recommender Systems (RecSys '11), Chicago, USA, p. 305-308.

Gianotti, R. C., Cazella, S. C. e Behar, P. A. (2019). A Model for Integrating Personality Traits into an Educational Recommender System. In: IEEE 19th International Conference on Advanced Learning Technologies (ICALT), Maceió, Brasil, p. 383-385.

Han, J., Kamber, M. e Pei, J. (2012). 8 - Classification: Basic Concepts. In: Data Mining: Concepts and Techniques (Third Edition) - A volume in The Morgan Kaufmann Series in Data Management Systems, p. 327-391. ISBN: 978-0-12-381479-1.

$\mathrm{Hu}, \mathrm{R}$. e Pu, P. (2011). Enhancing collaborative filtering systems with personality information. In: Proceedings of the Fifth ACM Conference on Recommender Systems (RecSys '11), New York, USA, p. 197-204.

IBM. (2019). IBM Cloud Docs - Personality Insights. Disponível em: http://cloud.ibm.com/docs/personality-insights. Acesso em: 08 jul. 2020.

Jaques, P. A. e Nunes, M. A. S. N. (2019). Computação afetiva aplicada à educação. In: Pimentel, M., Sampaio, F. F. e Santos, E. O. (Org.). Informática na Educação: técnicas e tecnologias computacionais. Porto Alegre: Sociedade Brasileira de Computação. (Série Informática na Educação, v. 3.).

Katarya, R. e Verma, O. P. (2016). Recent developments in affective recommender systems. Physica A: Statistical Mechanics and its Applications, v. 461, p. 182-190.

Klašnja-Milićević, A., et al. (2017). E-Learning Systems: Intelligent Techniques for Personalization. Springer. ISBN: 978-3-319-41163-7.

Laisa, J., et al. (2018). Uma Revisão Sistemática da Literatura sobre Sistemas de Recomendação Educacional. In: Anais do IX Computer on the Beach, Florianópolis, p. 751-760.

Leite, R., et al. (2019). Sistemas de Recomendação em Ambientes Educacionais: Estado da Arte 
IX Congresso Brasileiro de Informática na Educação (CBIE 2020)

Anais do XXXI Simpósio Brasileiro de Informática na Educação (SBIE 2020)

e Perspectivas Futuras. In: Anais dos Workshops do Congresso Brasileiro de Informática na Educação, v. 8, n. 1, p. 109-118.

Magalhães, J., et al. (2015). Recommending Scientific Papers: Investigating the User Curriculum. In: Proceedings of the Twenty-Eighth International Florida Artificial Intelligence Research Society Conference.

Moraes, T. C. H. e Stiubiener, I. (2019). Sistemas Híbridos para Recomendações Educacionais: Uma Revisão Sistemática da Literatura. In: Anais do Simpósio Brasileiro de Informática na Educação (SBIE), Brasília, p. 1331-1340.

Morais, F., et al. (2017). Computação Afetiva aplicada à Educação: uma revisão sistemática das pesquisas publicadas no Brasil. In: Anais do Simpósio Brasileiro de Informática na Educação (SBIE), Recife, p. 163-172.

Nunes, M. A. S. N. e Hu, R. (2012). Personality-based recommender systems: an overview. In: Proceedings of the Sixth ACM Conference on Recommender Systems (RecSys '12), ACM, New York, USA, p. 5-6.

Pinho, P. C. R., et al. (2019). Developments in Educational Recommendation Systems: a systematic review. IEEE Frontiers in Education Conference (FIE), Covington, KY, USA, 2019, p. 1-7.

Reis, R. C. D., et al. (2018). Affective states in computer-supported collaborative learning: Studying the past to drive the future. Computers \& Education, v. 120, p. 29-50.

Roshchina, A., Cardiff, J. e Rosso, P. (2015). TWIN: Personality-based Intelligent Recommender System. Journal of Intelligent \& Fuzzy Systems, IOS Press, v. 28, n. 5, p. 2059-2071.

Salehi, M. (2014). Latent Feature Based Recommender System for Learning Materials Using Genetic Algorithm. Journal of Information Systems and Telecommunication, v. 2, n. 3, p. 137-144.

Schwartz, H. A., et al. (2013). Personality, Gender, and Age in the Language of Social Media: The Open-Vocabulary Approach. PLoS ONE, v. 8, n. 9, p. e73791.

Shah, D., Shah, P. e Banerjee, A. (2017). Similarity based regularization for online matrixfactorization problem: An application to course recommender systems. In: TENCON 20172017 IEEE Region 10 Conference, p. 1874-1879.

Su, C.-Y., et al. (2015). A Cluster-based Personalized Item Recommended Approach on the Educational Assessment System. International Journal of Emerging Technologies in Learning (iJET), v. 10, n. 5, p. 52-58.

Tkalčič, M. (2018). Emotions e personality in recommender systems: tutorial. In: Proceedings of the 12th ACM Conference on Recommender Systems (RecSys'18), Vancouver, ACM, New York, USA, p. 535-536.

Tkalčič, M., et al. (2009). Personality Based User Similarity Measure for a Collaborative Recommender System. In: Proceedings of the 5th Workshop on Emotion in HumanComputer Interaction-Real World Challenges, p. 30-37.

Vygotsky, L. (1978). Mind in Society: The Development of Higher Psychological Processes. Harvard University Press, Cambridge, MA.

Xia, F., et al. (2014). Socially Aware Conference Participant Recommendation with Personality Traits. IEEE Systems Journal, p. 1-12.

Yakhchi, S., et al. (2020). Enabling the Analysis of Personality Aspects in Recommender Systems. arXiv preprint arXiv:2001.04825.

Zarzour, H., et al. (2018). A new collaborative filtering recommendation algorithm based on dimensionality reduction and clustering techniques. In: 9th International Conference on Information and Communication Systems (ICICS), IEEE, p. 102-106. 